\title{
Improved plaque neovascularization following 2-year atorvastatin therapy based on contrast-enhanced ultrasonography: A pilot study
}

\author{
BIN XU $^{1 *}$, JIN XING $^{2 *}$, WENQING WU ${ }^{3 *}$, WEN-JING ZHANG ${ }^{1}$, QIAN-QIAN ZHU ${ }^{1}$, DAN ZHANG ${ }^{2}$, \\ NAN-NAN SUN ${ }^{1}$, CHAN WU $^{1}$, GENG-JIE KANG ${ }^{1}$, LIN ZHAI $^{2}$, WEI-DONG LI ${ }^{1}$, YAN MENG ${ }^{2}$ and TIE-YING DU \\ Departments of ${ }^{1}$ Neurology and ${ }^{2}$ Ultrasound, Fuxing Hospital, Capital Medical University, Beijing 100038; \\ ${ }^{3}$ Department of Neurology, Beijing Ditan Hospital, Capital Medical University, Beijing 100015, P.R. China
}

Received May 25, 2016; Accepted April 7, 2017

DOI: $10.3892 /$ etm.2018.5926

\begin{abstract}
The present study assessed changes in carotid plaque neovascularization following long-term atorvastatin therapy (20 mg/day) using contrast-enhanced ultrasonography (CEUS). In this prospective case series, seven males (mean age, 68 99 years) and three females (mean age, $67 \pm 10$ years) with a total of 13 carotid plaques underwent standard ultrasonography and CEUS at baseline, as well as after 1 and 2 years of atorvastatin treatment. The same plaques were then examined using real-time CEUS. The results of the enhanced intensity of plaque neovascularization at baseline were compared with results obtained during follow-up to examine the effects of long-term atorvastatin therapy. Standard ultrasonography revealed that 7 of the 13 carotid plaques were uniformly echolucent, whereas 6 carotid plaques were predominantly echolucent. CEUS revealed an enhanced intensity of 10.5 \pm 2.1 decibels (dB) prior to treatment, which decreased significantly to $7.3 \pm 2.6 \mathrm{~dB}$ following 2 years atorvastatin therapy $(\mathrm{P}<0.001)$. The ratio of enhanced intensity in the carotid artery lumen to that in the plaque was $3.10 \pm 1.10$ at baseline and this value significantly increased to $4.96 \pm 2.98$ following treatment for 2 years $(\mathrm{P}<0.001)$. The current pilot study therefore indicates that two-year atorvastatin therapy ( $20 \mathrm{mg} /$ day) may reduce plaque neovascularization in the Chinese population.
\end{abstract}

Correspondence to: Professor Bin Xu, Department of Neurology, Fuxing Hospital, Capital Medical University, 20 Fu Xing Men Wai Avenue, Xicheng, Beijing 100038, P.R. China

E-mail:binxudoc@163.com

${ }^{*}$ Contributed equally

Key words: carotid artery atherosclerosis, vulnerable plaque, contrast-enhanced ultrasonography, neovascularization, statins

\section{Introduction}

Vulnerable plaques are plaques that are more likely to rupture and cause cerebrovascular events. The likelihood that a plaque will become vulnerable depends on a number of factors including the degree of stenosis, plaque morphology and plaque pathophysiology, such as intraplaque hemorrhage (1-4). Angiogenesis is important in determining plaque development and vulnerability. Plaque neovascularization is more extensive in symptomatic and vulnerable carotid artery plaques $(5,6)$ and immunohistochemical studies in humans have confirmed that the increased density of microvessels is associated with plaque rupture $(7,8)$. It has been demonstrated that vessel density is two times higher in vulnerable plaques than in stable plaques, which results in severe luminal narrowing. Furthermore, vessel density is up to four times higher in ruptures than in stable plaques (9).

It would be ideal to develop a noninvasive method capable of analyzing intraplaque angiogenesis and assessing whether these plaques are vulnerable to rupture. Sophisticated techniques, including computerized tomography angiography with contrast agents and positron emission tomography, have been developed to perform carotid artery plaque imaging in vivo $(10,11)$. However, these techniques are expensive and require substantial exposure to radiation, making them unsuitable for use during routine follow-up. Furthermore, they are unable to identify neovascularization. Although magnetic resonance imaging can determine the neovascularity of plaques, it requires the use of expensive apparatus and operation by trained practitioners. This is not feasible in many parts of the world. Standard ultrasonography can provide information on plaque morphology based on ultrasonic echolucency (12) but is inadequate for assessing plaque neovascularization (13). Due to these challenges, no imaging technique has been established as a 'gold standard' for analyzing vulnerable carotid plaques (14) and little is known about how inflammation or morphological changes in such plaques lead to cerebrovascular events.

Contrast-enhanced ultrasonography (CEUS) is a promising noninvasive tool for the visualization of plaque neovascularization. It combines the high spatial and temporal 
resolution of standard vascular ultrasonography with the properties of contrast agent microbubbles, which behave as intravascular tracers (15). CEUS reveals plaque biological activity and vascularization in vivo and a number of studies have suggested that CEUS may be useful for plaque risk stratification and assessing atherosclerosis progression and regression $(13,16,17)$. However, to the best of our knowledge, no studies have directly compared the effectiveness of CEUS with standard ultrasonography for assessing plaque neovascularization in patients at risk of atherosclerosis.

Statins are widely used to reduce cholesterol levels in patients at risk of atherosclerosis and changes in carotid plaques revealed by ultrasound are usually observed following large doses of atorvastatin ( 80 or $40 \mathrm{mg} /$ day) (18). Few studies have examined the effects of small doses of statins administered over a long time on carotid artery neovascularization. Thus the current study used CEUS to determine whether carotid plaque echogenicity and intra-plaque neovascularization were decreased following two-year atorvastatin therapy (20 mg/day) in Chinese patients.

\section{Patients and methods}

Patients. Patients scheduled to undergo standard ultrasonography of the carotid artery in the Department of Ultrasound, Fuxing Hospital (Beijing, China) between March 2009 and May 2012 were recruited. Among the 62 patients initially recruited, 10 patients ( 7 male patients and 3 female patients) were included in the current study.

Patients were eligible as long as they had at least one atherosclerotic plaque in the carotid artery that was thicker than $2.0 \mathrm{~mm}$ (19) and which was determined to be uniformly or predominantly echolucent by standard ultrasonography. Atherosclerotic plaques were defined according to the Mannheim consensus, which was the presence of focal structures encroaching into the arterial lumen by $>0.5 \mathrm{~mm}$, by $50 \%$ of the thickness of the surrounding intima-media complex or by the thickness of the intima-media layer if this was $>1.5 \mathrm{~mm}$ (20). Patients were excluded from the current study if they had known allergies to albumin or to standard ultrasonography contrast medium.

Each patient was administered atorvastatin calcium tablets (Pfizer Pharmaceutical Co., Ltd., Dalian, China; 20 mg/day taken orally once a day) for 2 years and all patients were able to continue their medications throughout. The dose of $20 \mathrm{mg} /$ day was selected as the majority of Chinese people do not have high cholesterol levels; levels of $<1.04 \mathrm{mmol} / 1$ are very common (21). In addition, as atorvastatin induces side effects in the liver, many patients are unable to tolerate higher doses. The current prospective pilot study was approved by the Research Ethics Committee of Fuxing Hospital and written informed consent was obtained from each patient.

Analysis of carotid plaques. All subjects were analyzed using standard ultrasonography and CEUS at baseline prior to initiation of atorvastatin therapy and the same examination was performed following 1 and 2 years of treatment. Standard ultrasonography and CEUS were performed using an Acuson Sequoia 512 ultrasound machine (Siemens Medical Solutions; Mountain View, CA, USA) equipped with an 8 -L probe and operated at a transmission frequency of 8-15 MHz. These procedures were performed by a trained vascular technologist who was blinded to the history of the participant. Prior to re-examination, the technologist reviewed the previous examination results of each patient to ensure that the same plaques were assessed that had been assessed previously.

As each patient lay in the supine position, the left and right carotid arteries were examined with the head supported at a $45^{\circ}$ angle and turned to the contralateral side. The common carotid artery, extracranial segments of the internal carotid artery and external carotid artery were examined in the longitudinal and transverse planes using standard ultrasonography. Maximal plaque thickness was measured as maximal intima-media thickness. Uniformly or predominantly echolucent lesions with plaques thicker than $2.0 \mathrm{~mm}$ were recorded during standard ultrasonography and CEUS, and were analyzed later.

Following standard ultrasonography, the same plaques were examined using real-time CEUS. Coded pulse inversion imaging was activated, image contrast was maximized and the mechanical index was reduced to 0.18-0.35. Using a region below the plaques of interest, the technician adjusted the time gain compensation to achieve homogeneous signal intensity for the carotid artery, while minimizing noise from the carotid artery wall and the plaque. All settings were kept constant throughout each examination.

CEUS imaging was performed following injection of the intravascular tracer SonoVue (Bracco SpA, Milan, Italy), consisting of sulfur hexafluoride phospholipid-stabilized microbubbles with a mean diameter of $2.5 \mu \mathrm{m}$ and a concentration of $1-5 \times 10^{8} / \mathrm{ml}(22)$. The microbubbles could freely flow through the tissues of tiny capillaries. However, they could not enter the tissue space through the vascular endothelial cells, which is a perfusion area limited in the vascular bed and is not involved in the outer region (23). Prior to use, $5 \mathrm{ml}$ saline solution was added to the lyophilized powder under a sulfur hexafluoride atmosphere and shaken thoroughly prior to use. The contrast agent was injected via the antecubital vein as a $2.2 \mathrm{ml}$ bolus within $2-3 \mathrm{sec}$, followed by a $5 \mathrm{ml}$ saline bolus. The appearance of the contrast effect was observed inside the lumen of the carotid artery 15-30 sec following the injection. A contrast-enhanced carotid cineloop was acquired starting at least $3 \mathrm{sec}$ prior to injection of the contrast material and this ended 5 min following the appearance of the contrast effect in the carotid artery lumen. Videos were digitally stored for later analysis. Participants were observed for $30 \mathrm{~min}$ before they were allowed to leave, in case any complications developed.

Analysis of standard ultrasound images. Maximal plaque thickness was measured from the media-adventitia to the intima-lumen boundaries and determined as the maximal intima-media thickness in a longitudinal image. Echolucent and mixed plaques were analyzed. Homogeneous echo plaques were defined as plaques with an echogenicity less than that of the surrounding adventitia for $>80 \%$ of the plaque area, without acoustic shadowing. Mixed plaques were defined as plaques containing $<90 \%$ of the circumferential calcification or with associated echo-dense and anechoic regions that 
occupied $<80 \%$ of the plaque area (24). Homogeneous echo plaques were named as 1 and mixed as 2 .

The dynamics of echogenic reflections from microspheres in the lumen of the carotid artery and intraplaque microvessels were observed. Subsequently, the region of interest (ROI), a circle constructed within the interested region to generate the time-intensity curve, was determined within the plaque (ROI-P) as a whole and in the lumen of the carotid artery near the plaque (ROI-L).

Plaque enhancement was quantified offline using the time-signal intensity curve analysis software (Research-Arena; Unterschleissheim, Germany). installed on the Acuson Sequoia 512. This software is able to exhibit the signal intensity-time curve in ROIs during enhancement. The following time-intensity curve parameters were noted: Baseline intensity (BI), arrival time (AT), time to peak (TTP) and peak intensity (PI). Due to the ultrasound contrast agent, the intra-plaque signal intensity increased. Thus, enhanced intensity (EI) was calculated as follows: $\mathrm{EI}=\mathrm{PI}-\mathrm{BI}$. EI is a parameter that measures the intensity differences between pre- and post-injections of the intravascular tracer, SonoVue, within the plaque ROI. Relative plaque enhancement (EI-R), measured at the separate peak enhancement point in the blood and plaque, was calculated as the ratio of enhanced intensity in the carotid artery lumen (EI-L) to the enhanced intensity in the plaque (EI-P) using the following formula: EI-R=EI-L/EI-P.

Quantitative data were retrospectively and independently analyzed by two investigators, who were blinded to the identity of the patient. Any disagreements were resolved by discussion.

Statistical analysis. Values were reported as mean \pm standard deviation, where appropriate. Data analysis was performed using SPSS 16.0 (SPSS, Inc., Chicago, IL, USA). Repeated-measurement analysis of variance (ANOVA) was used to compare CEUS parameters at baseline and after one and two years of atorvastatin treatment. Post-hoc statistical tests (Bonferroni test) were performed after ANOVA to account for the multiple comparisons. $\mathrm{P}<0.05$ was considered to indicate a statistically significant difference.

\section{Results}

Patients. During the study period, 10 consecutive subjects were enrolled: 7 males with a mean age of $68 \pm 9$ years and 3 females with a mean age of $67 \pm 10$ years (Table I). The number of patients with different risk factors (diabetes mellitus, hypertension, smoking history, coronary artery disease, stroke and peripheral arterial disease) were as follows: 8 patients had diabetes mellitus, 10 patients had hypertension, 5 patients had an active smoking history, 8 patients had coronary artery disease, 10 patients had experienced stroke and 1 patient had peripheral arterial disease. All patients presented with nonspecific neurologic symptoms including vertigo and syncope. The 10 patients included in the current study presented with a total of 13 carotid plaques.

Blood lipid levels. A total of $4 \mathrm{ml}$ blood was drawn using BD Vacationer vacuum blood collection tubes (Suzhou BD Medical Devices Co., Ltd) and blood lipid parameters (total cholesterol, triglycerides, high-density lipoprotein
Table I. Clinical characteristics of patients.

\begin{tabular}{lc}
\hline Characteristics & Number \\
\hline Age (years) & $68.9 \pm 9.2$ \\
Sex & \\
Male & 7 \\
Female & 3 \\
Diabetes & 8 \\
Hypertension & 10 \\
Smoking & 5 \\
Clinical history & \\
Coronary artery disease & 8 \\
Stroke & 10 \\
Peripheral arterial disease & 1 \\
\hline
\end{tabular}

Age is presented as the mean $\pm \mathrm{SD}$.

cholesterol and low-density lipoprotein cholesterol) were measured using an analyzer (Beijing Zhou Tian Hua Feng Medical Instruments Co. Ltd.) to detect blood fat at baseline and 1 and 2 years following atorvastatin treatment. All blood lipid indexes are presented in Table II, which was simultaneously checked during the ultrasound examination. From these results, it could be observed that there were no significant differences in blood lipid parameters among any of the different time points.

Ultrasound examination for carotid artery plaques. For each of the plaques, standard ultrasonography was used to evaluate lesion echogenicity, while CEUS was used to perform the visual and quantitative analysis of neovascularization. Each technique was applied at baseline (at the time of study enrollment) and following 1 and 2 years of atorvastatin treatment.

At baseline, standard ultrasonography revealed seven uniformly echolucent lesions and six predominantly echolucent lesions. Following atorvastatin therapy for 1 year, the same technique revealed that all plaques were predominantly echogenic. Following 2 years of therapy, all plaques appeared uniformly echogenic or extensively calcified (Table III).

The carotid plaque sizes at pre- and post-atorvastatin therapy are presented in Table IV. ANOVA indicated a significant difference in carotid plaque size between pre- and post-atorvastatin therapy $(\mathrm{P}=0.016)$. These results demonstrate that carotid plaque size shrunk each year following atorvastatin therapy.

CEUS analysis at baseline revealed an average EI-P of $10.55 \pm 2.08$ decibels $(\mathrm{dB})$ and an average EI-R of $3.11 \pm 1.08$ for all 13 plaques. Following 1-year atorvastatin therapy, EI-P decreased to $8.96 \pm 2.80 \mathrm{~dB}$, while EI-R increased to $3.61 \pm 1.33$. Following 2 years of therapy, these values were $7.27 \pm 2.57 \mathrm{~dB}$ and $4.96 \pm 2.99$, respectively. Values at both follow-ups differed significantly from those at baseline (Table IV, Fig. 1).

In comparison with the baseline, average EI-P decreased and EI-R increased after atorvastatin therapy 1 year and 2 years (Tables $\mathrm{V}$ and $\mathrm{VI}$ ). 
Table II. Blood lipid parameters.

\begin{tabular}{|c|c|c|c|c|}
\hline Variable & Baseline & 1 year & 2 years & P-value \\
\hline TCHO (mmol/l) & $3.40 \pm 0.21$ & $3.19 \pm 0.28$ & $3.37 \pm 0.24$ & 0.540 \\
\hline TG (mmol/l) & $1.69 \pm 0.42$ & $1.52 \pm 0.37$ & $1.40 \pm 0.20$ & 0.166 \\
\hline HDL-C (mmol/l) & $1.16 \pm 0.10$ & $1.05 \pm 0.08$ & $1.11 \pm 0.09$ & 0.673 \\
\hline LDL-C (mmol/l) & $2.14 \pm 0.73$ & $2.09 \pm 0.87$ & $2.16 \pm 0.69$ & 0.695 \\
\hline
\end{tabular}

All data are presented as the mean \pm SD. TC, total cholesterol; TG, triglyceride; HDL-C, high-density lipoprotein cholesterol; LDL-C, low-density lipoprotein cholesterol.

Table III. Carotid plaque echogenicity features.

\begin{tabular}{lccc}
\hline Echogenicity features & Baseline & 1 year & 2 years \\
\hline Homogeneous Echo Plaque & 7 & 4 & 0 \\
Mixed Plaque & 6 & 9 & 13 \\
Total & 13 & 13 & 13 \\
\hline
\end{tabular}

Fig. 2 presents a patient who had a large plaque in the common carotid artery that was identified by computed tomography angiography. CEUS and time-signal intensity curves analysis revealed enhanced intensity in the plaque and lumen. After 2 years of treatment, the echo of the plaques was extensively enhanced by calcification, and the plaque neovascularization in the carotid plaques decreased.

\section{Discussion}

In the current pilot study, it was identified that CEUS is superior to standard ultrasonography at measuring plaque neovascularization in patients at risk of atherosclerosis. Since poor neovascularization is associated with plaque vulnerability, CEUS may provide a noninvasive method for assessing the risk of cerebrovascular events. CEUS also determined that 2-year atorvastatin (20 mg/day) therapy is able to significantly reduce neovascularization, suggesting that it induces a plaque-stabilizing effect.

A previous study has detected change in carotid plaques by ultrasound following administration of 40 or $80 \mathrm{mg} /$ day atorvastatin in Western populations, and the administration of $20 \mathrm{mg} /$ day atorvastatin as a homeopathic dose appeared to induce no strong response in the plaques in Western populations (25). However, some studies have indicated that small doses of atorvastatin are safe and effective to administer to ethnic Chinese patients $(21,26)$. Colhoun et al $(27)$ reported that $10 \mathrm{mg} /$ day atorvastatin was safe and effective at reducing the risk of first cardiovascular events including stroke in the UK and Ireland with type II diabetes, without elevating low-density lipoprotein (LDL) cholesterol levels. This may be due to ethnic differences. Studies have confirmed that $20 \mathrm{mg} /$ day atorvastatin reduces LDL levels, as well as inflammation and thrombogenesis, in Chinese patients with acute ischemic stroke caused by large artery atherosclerosis $(21,26,28-30)$. A possible reason for the greater effect of

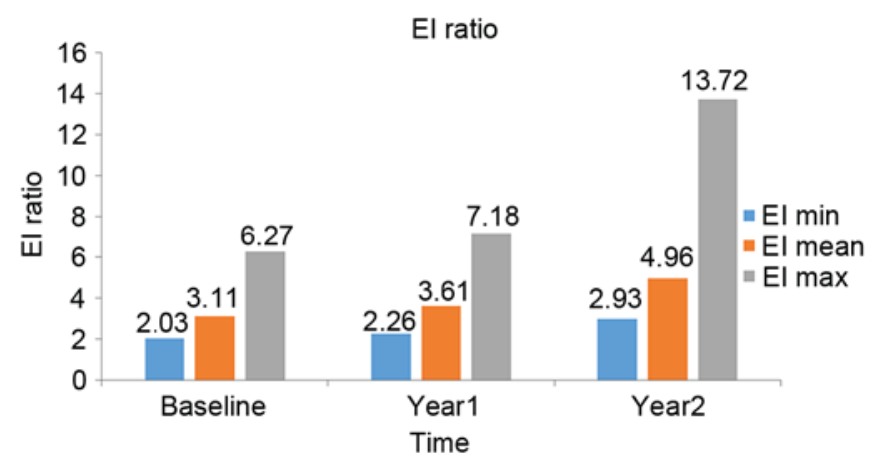

Figure 1. EI ratios were determined at durations of atorvastatin calcium tablets $20 \mathrm{mg} / \mathrm{kg}$ via oral administration once daily for 2 years. EI ratio following 2 years of atorvastatin treatment was higher than that of the baseline and 1 year of treatment. EI, enhanced intensity; EI ratio, enhanced intensity in the carotid artery lumen to that of in the plaque; EI, enhanced intensity.

moderate statin doses in Asian compared with Western populations may be the difference in statin pharmacokinetics (31). In addition, a previous study indicated that a double dose or increased statin administration did not bring significant clinical effectiveness (32). In addition, the results of the CHILLAS study may be considered a representative of what can be achieved by lipid-lowering treatment (29). In Taiwan, the PAPAGO-T study conducted among high-risk patients including those with type II diabetes mellitus revealed that $10 \mathrm{mg}$ /day atorvastatin was well-tolerated, lowered LDL-C levels and improved the lipid profile to a comparable degree in high-risk ethnic Chinese patients with hypercholesterolemia (33).

Plaque inflammation and neovascularization are histological markers of vulnerable plaques and predictors of unstable atherosclerotic lesions in patients with cerebro- or cardiovascular disease (17). This has led to increasing interest in the inflammatory and histological processes that occur in atherosclerotic lesions and give rise to cerebrovascular events $(34,35)$. A noninvasive method capable of evaluating plaque neovascularization is required to evaluate these processes. The microbubbles used with CEUS in the current study could freely move through the tissues of tiny capillaries but could not enter the tissue space through vascular endothelial cells. Hence, CEUS was used to assess neovascularity in a noninvasive manner.

Homogeneous echo plaques on standard ultrasonography B-mode images reflect the histological features of plaque 
Table IV. Carotid plaque sizes and contrast enhancement.

\begin{tabular}{lcccc}
\hline Variable & Baseline & 1 year & 2 years & P-value \\
\hline Plaque sizes $\left(\mathrm{mm}^{2}\right)$ & $40.98 \pm 15.94$ & $29.58 \pm 12.75$ & $24.57 \pm 13.33$ & $0.016^{\mathrm{a}}$ \\
EI-P $(\mathrm{dB})$ & $10.55 \pm 2.08$ & $8.96 \pm 2.80$ & $7.27 \pm 2.57$ & $<0.001^{\mathrm{a}}$ \\
$\Delta$ TTP $(\mathrm{sec})$ & $2.20 \pm 1.70$ & $3.45 \pm 1.59$ & $3.62 \pm 1.60$ & $0.011^{\mathrm{a}}$ \\
$\Delta$ AT $(\mathrm{sec})$ & $1.44 \pm 1.22$ & $2.69 \pm 2.11$ & $3.20 \pm 2.07$ & 0.062 \\
EI-R & $3.11 \pm 1.08$ & $3.61 \pm 1.33$ & $4.96 \pm 2.99$ & $0.022^{\mathrm{a}}$
\end{tabular}

All data are presented as the means $\pm \mathrm{SD}$. ${ }^{\mathrm{a}} \mathrm{P}<0.05$ vs. other two groups. $\mathrm{dB}$, decibel; EI-P, enhanced intensity in plaque; EI-R, enhanced intensity ratio; AT, arrival time; TTP, time to peak.

Table V. Multiple comparisons of enhanced intensity in the plaque at different time points.

\begin{tabular}{lccc}
\hline & \multicolumn{3}{c}{ P-value } \\
\cline { 2 - 4 } Time point & Baseline & 1 year & 2 years \\
\hline Baseline & - & 0.002 & 0.000 \\
1 year & 0.002 & - & 0.012 \\
2 year & 0.000 & 0.012 & - \\
\hline
\end{tabular}

Table VI. Multiple comparisons of enhanced intensity in the plaque ratio at different time points.

\begin{tabular}{lccc}
\hline & \multicolumn{3}{c}{ P-value } \\
\cline { 2 - 4 } Time point & Baseline & 1 year & 2 years \\
\hline Baseline & - & 0.007 & 0.032 \\
1 year & 0.007 & - & 0.079 \\
2 year & 0.032 & 0.079 & - \\
\hline
\end{tabular}

instability. Such plaques are prone to rupture due to increased lipid content, macrophage density and intraplaque hemorrhage. Homogeneous echo plaques are also associated with an increased risk of ischemic stroke $(12,35)$. Standard ultrasonography of the patients in the current study at baseline revealed either uniform or predominant echolucent lesions. Following 2 years atorvastatin therapy, plaques became uniformly echogenic or extensively calcified, suggesting a lower risk of rupture. Furthermore, the size of the carotid plaques shrunk each year following atorvastatin therapy, suggesting that atorvastatin may inhibit the growth of plaques. However, the carotid plaque size shrunk less during the second year of therapy, compared with the decrease observed over the first year. This may be explained by the fact that when blood drug concentrations reach a certain degree following long-term atorvastatin therapy, patients develop a degree of tolerance to the drug. However, plaque stability does not appear to be associated with blood lipid levels and a previous study confirmed that blood lipid levels were not linearly associated with the stability of the plaque (36). CEUS analysis through the same treatment period has been proven to be effective at assessing neovascularization, and determined that atorvastatin significantly reduced EI-P and increased EI-R. EI-R is a straightforward ratio of signal intensities within the plaque ROI and the artery lumen at the time of peak intensity in the artery lumen. This ratio was selected as it can cancel the interference factor of different patients and represent the absolute value of enhancement $(37,38)$.

The results of the current study demonstrate the usefulness of CEUS, supporting the results of previous studies suggesting that this method is a promising noninvasive tool to visualize plaque neovascularization $(12,36)$. Shah et al $(35)$ identified a strong correlation between CEUS analysis of plaque neovascularization in carotid arteries and histological scores on surgical specimens. Coli et al (39) demonstrated that CEUS measurements of contrast enhancement correlated well with histologically determined neovessel density in plaques.

The results of the current study provide some of the first direct evidence that prolonged statin therapy reduces plaque neoangiogenesis. Angiogenesis in plaques may be triggered by hypoxia and inflammation $(40,41)$ and it has been demonstrated that statins reduce inflammation as well as lowering lipid levels $(42,43)$. Therefore, the anti-inflammatory effects of statins may mediate their therapeutic effect on plaque neovascularization. Larger controlled trials are required to validate CEUS as a routine screening and monitoring method for patients at risk of atherosclerosis. Such studies should also examine the mechanism of statin action in more detail.

In conclusion, the current pilot study suggests that CEUS is a promising potential surrogate end point in clinical trials that examine risk factors and treatments for atherosclerosis. Using this technique, it was suggested that long-term atorvastatin therapy may reduce plaque neovascularization and thereby reduce the risk of cerebrovascular events occurring.

\section{Acknowledgements}

The authors are grateful to Dr Lu Zhao Ling, for providing technical assistance. The present study was funded by the Chinese Medical Association Special Fund as part of the project 'Neovascularization within carotid atherosclerotic plaques with contrast-enhanced US angiography: Clinical Research' (grant no. 09010410196) and by the Beijing Natural 


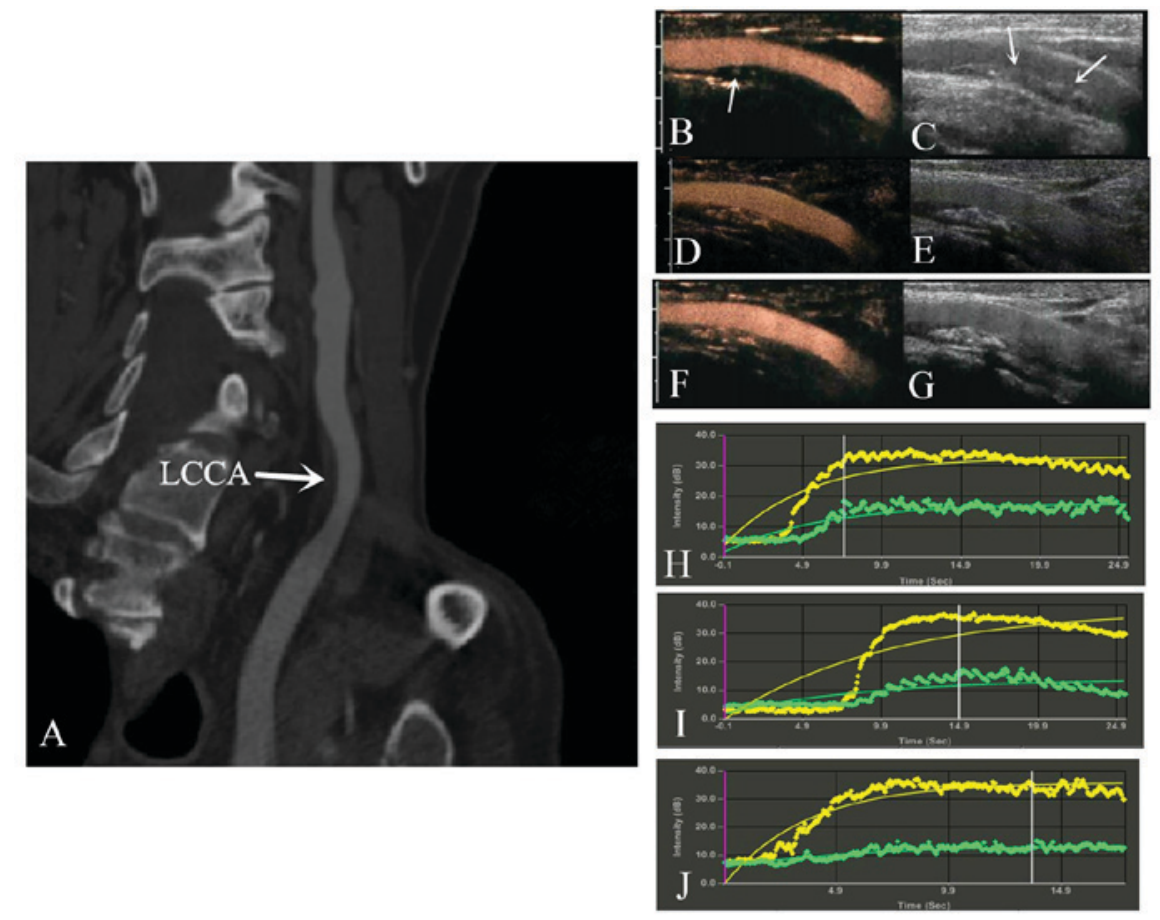

Figure 2. (A-J) Computed tomographic angiography analysis of the left common carotid artery. (A) A large soft plaque was indicated in the left common carotid artery (white arrow). (B) View following injection of contrast agent: The contrast agent entered into the plaque (white arrow) and (H) the time-signal intensity curves analysis revealed enhanced intensity in the plaque and lumen. (C) Longitudinal plane without contrast agent revealed a soft plaque in the CCA (white arrow). (D and E) Following 1 year of atorvastatin treatment, the plaque was enhanced without contrast agent and plaque neovascularization of CCA was reduced. (I) The time-signal intensity curves analysis revealed the arrive time which contrast agent entered into the plaque prolong and the peak intensity reduced slightly than before. ( $\mathrm{F}$ and $\mathrm{G}$ ) Following 2 years of atorvastatin treatment, the plaque was extensively enhanced without contrast agent and plaque neovascularization of CCA was reduced. (J) The time-signal intensity curves analysis revealed the arrive time which contrast agent entered into the plaque further prolong and the peak intensity reduced significantly. The lumen of the CCA was indicated by the yellow curve and plaque was indicated by the green curve in all time-signal intensity curves. CCA, common carotid artery; LCCA, left common carotid artery.

Science Foundation Program and Scientific Research Key Program of Beijing Municipal Commission of Education (Item No: KZ201510025031).

\section{References}

1. McCarthy MJ, Loftus IM, Thompson MM, Jones L, London NJ, Bell PR, Naylor AR and Brindle NP: Angiogenesis and the atherosclerotic carotid plaque: An association between symptomatology and plaque morphology. J Vasc Surg 30: 261-268, 1999.

2. Finn AV, Nakano M, Narula J, Kolodgie FD and Virmani R: Concept of vulnerable/unstable plaque. Arterioscler Thromb Vasc Biol 30: 1282-1292, 2010.

3. Michel JB, Delbosc S, Ho-Tin-Noé B, Leseche G, Nicoletti A, Meilhac $\mathrm{O}$ and Martin-Ventura JL: From intraplaque haemorrhages to plaque vulnerability: Biological consequences of intraplaque haemorrhages. J Cardiovasc Med (Hagerstown) 13: 628-634, 2012.

4. Virmani R, Kolodgie FD, Burke AP, Finn AV, Gold HK, Tulenko TN, Wrenn SP and Narula J: Atherosclerotic plaque progression and vulnerability to rupture: Angiogenesis as a source of intraplaque hemorrhage. Arterioscler Thromb Vasc Biol 25: 2054-2061, 2005.

5. Di Stefano R, Felice F and Balbarini A: Angiogenesis as risk factor for plaque vulnerability. Curr Pharm Des 15: 1095-1196, 2009.

6. Sluimer JC and Daemen MJ: Novel concepts in atherogenesis: Angiogenesis and hypoxia in atherosclerosis. J Pathol 218: 7-29, 2009.

7. Dunmore BJ, McCarthy MJ, Naylor AR and Brindle NP: Carotid plaque instability and ischemic symptoms are linked to immaturity of microvessels within plaques. J Vasc Surg 45: 155-159, 2007.

8. Kodama T, Narula N, Agozzino M and Arbustini E: Pathology of plaque haemorrhage and neovascularization of coronary artery. J Cardiovasc Med (Hagerstown) 13: 620-627, 2012.
9. Chen S, Guo L, Chen B, Sun L and Cui M: Association of serum angiopoietin-1, angiopoietin-2 and angiopoietin-2 to angiopoietin-1 ratio with heart failure in patients with acute myocardial infarction. Exp Ther Med 5: 937-941, 2013.

10. Warburton L and Gillard J: Functional imaging of carotid atheromatous plaques. J Neuroimaging 16: 293-301, 2006.

11. Laufer EM, Winkens HM, Corsten MF, Reutelingsperger CP, Narula $\mathbf{J}$ and Hofstra L: PET and SPECT imaging of apoptosis in vulnerable atherosclerotic plaques with radiolabeled Annexin A5. Q J Nucl Med Mol Imaging 53: 26-34, 2009.

12. Grønholdt ML, Nordestgaard BG, Schroeder TV, Vorstrup S and Sillesen H: Ultrasonic echolucent carotid plaques predict future strokes. Circulation 104: 68-73, 2001.

13. Fabrizia Giannoni M, Vicenzini E, Monaco C and Cao P: Contrast enhanced ultrasonography and carotid plaque imaging: From the hemodynamic evaluation to the detection of neoangiogenesis-The new approach to the identification of the unstable plaque: From morphology to pathophysiology. In: Ultrasound Imaging. Tanabe M (ed). InTech, pp171-188, 2011.

14. Faggioli GL, Pini R, Mauro R, Pasquinelli G, Fittipaldi S, Freyrie A, Serra C and Stella A: Identification of carotid 'vulnerable plaque' by contrast-enhanced ultrasonography: Correlation with plaque histology, symptoms and cerebral computed tomography. Eur J Vasc Endovasc Surg 41: 238-248, 2011.

15. Feinstein SB: The powerful microbubble: From bench to bedside, from intravascular indicator to therapeutic delivery system, and beyond. Am J Physiol Heart Circ Physiol 287: H450-H457, 2004.

16. Feinstein SB: Contrast ultrasound imaging of the carotid artery vasa vasorum and atherosclerotic plaque neovascularization. J Am Coll Cardiol 48: 236-243, 2006.

17. Staub D, Patel MB, Tibrewala A, Ludden D, Johnson M, Espinosa P, Coll B, Jaeger KA and Feinstein SB: Vasa vasorum and plaque neovascularization on contrast-enhanced carotid ultrasound imaging correlates with cardiovascular disease and past cardiovascular events. Stroke 41: 41-47, 2010. 
18. Della-Morte D, Moussa I, Elkind MS, Sacco RL and Rundek T: The short-term effect of atorvastatin on carotid plaque morphology assessed by computer-assisted gray-scale densitometry: A pilot study. Neurol Res 33: 991-994, 2011.

19. Touboul PJ, Hennerici MG, Meairs S, Adams H, Amarenco P, Desvarieux M, Ebrahim S, Fatar M, Hernandez Hernandez R, Kownator S, et al: Mannheim intima-media thickness consensus. Cerebrovasc Dis 18: 346-349, 2004.

20. Touboul PJ, Hennerici MG, Meairs S, Adams H, Amarenco P, Bornstein N, Csiba L, Desvarieux M, Ebrahim S, Hernandez Hernandez R, et al: Mannheim carotid intima-media thickness and plaque consensus (2004-2006-2011). An update on behalf of the advisory board of the 3rd, 4th and 5th watching the risk symposia, at the 13th, 15th and 20th European Stroke Conferences, Mannheim, Germany, 2004, Brussels, Belgium, 2006, and Hamburg, Germany, 2011. Cerebrovasc Dis 34: 290-296, 2012

21. Yang W, Lu J, Weng J, Jia W, Ji L, Xiao J, Shan Z, Liu J, Tian H, $\mathrm{Ji}$ Q, et al: Prevalence of diabetes among men and women in China. N Engl J Med 362: 1090-1101, 2010.

22. Kern R, Szabo K, Hennerici M and Meairs S: Characterization of carotid artery plaques using real-time compound B-mode ultrasound. Stroke 35: 870-875, 2004.

23. Ferrara KW, Merritt CR, Burns PN, Foster FS, Mattrey RF and Wickline SA: Evaluation of tumor angiogenesis with US Imaging, Doppler, and contrast agents. Acad Radiol 7: 824-839, 2000 .

24. Staub D, Partovi S, Schinkel AF, Coll B, Uthoff $H$ Aschwanden M, Jaeger KA and Feinstein SB: Correlation of carotid artery atherosclerotic lesion echogenicity and severity at standard US with intraplaque neovascularization detected at contrast-enhanced US. Radiology 258: 618-626, 2011.

25. Kadoglou NP, Sailer N, Moumtzouoglou A, Kapelouzou A, Gerasimidis T and Liapis CD: Aggressive lipid-lowering is more effective than moderate lipid-lowering treatment in carotid plaque stabilization. J Vasc Surg 51: 114-21, 2010.

26. Gupta M, Martineau P, Tran T, Després JP, Gaw A, de Teresa E Farsang C, Gensini GF, Leiter LA, Blanco-Colio LM, et al: Low-density lipoprotein cholesterol and high-sensitivity $\mathrm{C}$-reactive protein lowering with atorvastatin in patients of South Asian compared with European origin: Insights from the achieve cholesterol targets fast with atorvastatin stratified titration (ACTFAST) study. J Clin Pharmacol 52: 850-858, 2012.

27. Colhoun HM, Betteridge DJ, Durrington PN, Hitman GA, Neil HA, Livingstone SJ, Thomason MJ, Mackness MI, Charlton-Menys V and Fuller JH; CARDS investigators: Primary prevention of cardiovascular disease with atorvastatin in type 2 diabetes in the collaborative atorvastatin diabetes study (CARDS): Multicentre randomised placebo-controlled trial. Lancet 364: 685-696, 2004.

28. Min L, Shao S, Wu X, Cong L, Liu P, Zhao H and Luo Y: Anti-inflammatory and anti-thrombogenic effects of atorvastatin in acute ischemic stroke. Neural Regen Res 8: 2144-2154, 2013.

29. Zhao SP, Yu BL, Peng DQ and Huo Y: The effect of moderate-dose versus double-dose statins on patients with acute coronary syndrome in China: Results of the CHILLAS trial. Atherosclerosis 233: 707-712, 2014.

30. Jia W and Zhou L: Effect of $20 \mathrm{mg} /$ day atorvastatin: Recurrent stroke survey in Chinese ischemic stroke patients with prior intracranial hemorrhage. J Clin Neurol 9: 139-143, 2013.
31. Lee E, Ryan S, Birmingham B, Zalikowski J, March R, Ambrose H, Moore R, Lee C, Chen Y and Schneck D: Rosuvastatin pharmacokinetics and pharmacogenetics in white and Asian subjects residing in the same environment. Clin Pharmacol Ther 78: 330-341, 2005.

32. Zhao SP, Yu BL, Peng DQ and Huo Y: The effect of moderate-dose versus double-dose statins on patients with acute coronary syndrome in China: Results of the CHILLAS trial. Atherosclerosis 233: 707-712, 2014.

33. Liu PY, Lin LY, Lin HJ, Hsia CH, Hung YR, Yeh HI, Wu TC, Chen JY, Chien KL and Chen JW: Pitavastatin and atorvastatin double-blind randomized comparative study among high-risk patients, including those with type 2 diabetes mellitus, in Taiwan (PAPAGO-T Study). PLoS One 8: e76298, 2013.

34. Desai MY and Schoenhagen P: Emergence of targeted molecular imaging in atherosclerotic cardiovascular disease. Expert Rev Cardiovasc Ther 7: 197-203, 2009.

35. Shah F, Balan P, Weinberg M, Reddy V, Neems R, Feinstein M, Dainauskas J, Meyer P, Goldin $M$ and Feinstein SB: Contrast-enhanced ultrasound imaging of atherosclerotic carotid plaque neovascularization: A new surrogate marker of atherosclerosis? Vasc Med 12: 291-297, 2007.

36. Hong LF, Yan XN, Fan Y, Wu Q, Luo SH, Yang B and Li JJ: Is the ratio of apoB/apoA-1 the best predictor for the severity of coronary artery lesions in Chinese diabetics with stable angina pectoris? An assessment based on Gensini scores. J Geriatr Cardiol 12: 402-409, 2015.

37. Zhang Q, Li C, Han H, Dai W, Shi J, Wang Y and Wang W: Spatio-temporal quantification of carotid plaque neovascularization on contrast enhanced ultrasound: Correlation with visual grading and histopathology. Eur J Vasc Endovasc Surg 50: 289-296, 2015.

38. Hoogi A, Adam D, Hoffman A, Kerner H, Reisner S and Gaitini D: Carotid plaque vulnerability: Quantification of neovascularization on contrast-enhanced ultrasound with histopathologic correlation. AJR Am J Roentgenol 196: 431-436, 2011.

39. Coli S, Magnoni M, Sangiorgi G, Marrocco-Trischitta MM, Melisurgo G, Mauriello A, Spagnoli L, Chiesa R, Cianflone D and Maseri A: Contrast enhanced ultrasound imaging of intraplaque neovascularization in carotid arteries: Correlation with histology and plaque echogenicity. J Am Coll Cardiol 52: 223-230, 2008

40. Coll B, Nambi V and Feinstein SB: New advances in noninvasive imaging of the carotid artery: CIMT, contrast-enhanced ultrasound, and vasa vasorum. Curr Cardiol 12: 497-502, 2010.

41. Kolodgie FD, Narula J, Yuan C, Burke AP, Finn AV and Virmani R: Elimination of neoangiogenesis for plaque stabilization: Is there a role for local drug therapy? J Am Coll Cardiol 49: 2093-2101, 2007

42. Jain RK, Finn AV, Kolodgie FD, Gold HK and Virmani R: Antiangiogenic therapy for normalization of atherosclerotic plaque vasculature: A potential strategy for plaque stabilization. Nat Clin Pract Cardiovasc Med 4: 491-502, 2007.

43. Doyle B and Caplice N: Plaque neovascularization and antiangiogenic therapy for atherosclerosis. J Am Coll Cardiol 49: 2073-2080, 2007. 